\title{
Developing soft $x$-ray spectroscopy for in-situ characterization of nanocatalysts in catalytic reactions
}

Hui Zhang ${ }^{1,2}$, Wei-Cheng Wang ${ }^{2,3}$, Per-Anders Glans ${ }^{2}$, Yi-Sheng Liu ${ }^{2}$, Mukes

Kapilashrami ${ }^{2}$, Jen-Lung Chen ${ }^{2,3}$, Chinglin Chang ${ }^{3}$, Miquel Salmeron ${ }^{4,5}$, Carlos

Escudero ${ }^{4}$, Elzbieta $\mathrm{Pach}^{4}$, Anders Tuxen ${ }^{4}$, Mahati Chintapalli ${ }^{4}$, Sophie Carenco ${ }^{4}$, Xuhui Sun ${ }^{*, 1}$, Jinghua Guo*,2,6

${ }^{1}$ Institute of Functional Nano and Soft Materials Laboratory (FUNSOM), Soochow

University, Suzhou 215123, China

${ }_{2}^{2}$ Advanced Light Source, Lawrence Berkeley National Lab, Berkeley, CA 94720, USA

${ }^{3}$ Department of Physics, Tamkang University, Tamsui 251, Taiwan

${ }^{4}$ Materials Sciences Division Sciences Division, Lawrence Berkeley National Lab,

Berkeley, CA 94720, USA

${ }^{5}$ Department of Materials Science and Engineering, University of California, Berkeley

${ }^{6}$ Department of Chemistry and Biochemistry, University of California, Santa Cruz, CA 95064, USA

\begin{abstract}
:
Understanding the mechanisms of catalytic and reactions calls for in-situ/operando spectroscopic characterization. Here we report the developments of in-situ reaction cells at the Advanced Light Source for soft X-ray spectroscopic studies of nanoparticle catalysts during the catalytic reactions. The operation of these various cells and their capabilities are illustrated with examples from the studies of Co-based nanocatalysts.

*jguo@lbl.gov (JHG) and xhsun@suda.edu.cn (XHS)
\end{abstract}


Growing environmental concerns have sparked the renewed interest in the FischerTropsch process to synthesize cleaner chemical fuels from syngas, which are produced from abundant raw materials such as coal or biomass. Catalysis is a powerful technique for developing chemical processes to make efficient use of energy and raw materials, while achieving the minimal impact on the environment. The development of highly active and selective, low cost, and long-lifetime catalysts has been a major force for fuel production from alternative sources, and for the commodity chemical production from sustainable processes. [1]

Although the efficiency of catalytic processes has been improved in the past decades [2, 3], fundamental questions concerning the reaction mechanism and structural properties of the catalysts remain unsolved. While catalyst turnover frequency (a.k.a. reactivity) is an important characteristic for its efficiency and overall performance, catalytic reaction selectivity is the most desirable property. Development of the novel and high throughput synthesis routes for highly effective nanoparticles (NPs) with the controlled size, shape, and composition paves the path for another degree of freedom: selectivity. In addition to have a good control of the growth of the catalytic NPs, it is equally important to have access to state-of-the-art characterization tools (e.g. in-situ microscopy and spectroscopy tools [4]) to be able to locally probe and understand the reaction mechanisms for improved design and reaction efficiency of catalysts.

Understanding heterogeneous catalysis holds the key to improve industrial chemical processes that have traditionally been relying on a trial and error basis for further development and optimization of their performance. As for an example, cobalt is well known for its application in catalytic hydrogenation reactions of $\mathrm{CO}$ and $\mathrm{CO}_{2}$ for production of gaseous or liquid hydrocarbons, with a long history of producing synthetic fuels. However, till date the role of Co catalyst in the model oxidation reaction of $\mathrm{CO}$ to $\mathrm{CO}_{2}$ is still under debate.

The direct interaction to drive the surface science in ultrahigh vacuum (UHV) condition is firmly rooted in physics and the molecular side in chemistry [5]. Surface sensitive experimental techniques such as X-ray photoelectron spectroscopy (XPS) have been employed to investigate the oxidation states of catalysts in the catalytic reactions as well 
as the composition of the constituents in the reaction. XPS is powerful for studying heavy catalytic elements, such as $\mathrm{Pt}, \mathrm{Pd}, \mathrm{Ru}, \mathrm{Au}$, and $\mathrm{Ce}$ in which the d-levels are mostly relevant to the catalytic performance. Traditionally, this technique has required ultra-high vacuum (UHV) sample environment with controlled modeling systems to simulate the surface reaction of catalysis, thus the experimental condition is far from the industrial catalytic reaction conditions.

There have been extensive studies of the catalytic activity and selectivity towards different reactions of interest in Co NP catalysis (Fischer-Tropsch, Water-Gas Shift Reaction, $\mathrm{CO}$ oxidation, etc) [6-11]. The experimental findings provide new insights at a fundamental scientific level that can subsequently be transferred to the catalytic industrial applications. The underlying motivation for studying the active state and structure of e.g. NPs based catalysts in reactions under gas or liquid environments is the plausible existence of transient states. It is likely that the transient state is different from those detected in experiments performed ex situ (prior and/or post exposure to the reaction conditions, and then measured under UHV condition). The in-situ characterization instrumentation developed at the Advanced Light Source (ALS) allows a collection of core-level spectra more effectively in gaseous environments that mimic the operating conditions of catalytic systems, such as ambient-pressure XPS (AP-XPS, up to 10 torr of gas pressure) [12]. This state-of-the-art instrumentation can today be found in a number of synchrotron radiation facilities in the world. There are several companies who provide commercial setups of this kind to be used in the laboratories and synchrotron radiation facilities (e.g. SPECS, SCIENTA, etc.).

Hard X-ray absorption fine structure (XAFS) can reach the L-edges of heavy elements and also the K-edge of $3 \mathrm{~d}$ transition metal elements that are most commonly used in catalytic reactions, and has thus been employed to e.g. methanol oxydehydrogenation on polycrystalline surfaces under industrial reaction conditions [13-16]. Another active technique to study the nanostructured catalysts is soft X-ray absorption spectroscopy (XAS), which is more challenging for in-situ characterization due to its limited penetration depth (typically 100-200 nm). We will present some of the developments in in-situ soft X-ray spectroscopy at the ALS, which have allowed samples to be studied both in gas phases up to 1 bar pressure [17-19] and liquid phase [20, 21]. Such 
experimental developments have led to the study of $3 \mathrm{~d}$ transition metals as catalysts in catalytic reactions. For example XAS probes the electron excitation of the $3 \mathrm{~d}$ transition metals (e.g. Co) from the $2 p$ core level to unoccupied $3 d$ states, which provides critical information about the oxidation states and crystal structure of Co catalysts. Thus, the chemical and physical properties of Co catalysts during a catalytic reaction are revealed.

\section{Experimental Setup: the development of in-situ cells}

Soft X-ray spectroscopy such as XAS (in fluorescence detection mode) and resonant soft $\mathrm{X}$-ray emission spectroscopy (RXES) are essentially bulk sensitive, since the penetration depth of soft X-ray photons is about a few hundreds of nanometers in solid materials [22]. When membrane windows with considerable transmittance are utilized to separate the liquid or gas samples from the experimental vacuum environment, the soft X-ray spectroscopy experimental setup can then be used to study the gas and liquid samples or other samples, such as e.g. a gas/solid and liquid/solid interfaces in the conditions that are relevant for catalytic reaction studies. The in-situ cells developed at the ALS have undergone a number of modification/optimization steps for improved the performance. The first generation gas cell comprised of an "entrance" window for incoming soft Xrays ( $c a .0 .2 \mathrm{~mm}$ in diameter) and a slit-like "exit" window ( $c a .0 .5 \mathrm{~mm}$ wide and $15 \mathrm{~mm}$ long) for outgoing soft X-ray photons, respectively [19]. The dimensions of the "exit" window matches the detection geometry of the grating spectrometer and thus allows higher intensity to be measured as the interaction length between incoming X-ray and gas molecules can be extended at lower gas pressure (typically around 1 torr). This design enabled the studies of gas molecules, in which the XAS and RXES spectra were measured at gas pressure ranging from a few hundred millitorr to $c a$. 20 torr (depending on the focus on energy regions of $\pi^{*}$ or Rydberg states) [17, 18, 23-28]. Later developments made it possible to study the gaseous samples at higher gas pressures [29, 30]. There has been an increasing interest in studying the electronic structure of solidstate materials in a gas environment. For example, when hydrogen adsorption in the multilayers and superlattices gained increasing interest, it was possible to tailor the effective potential, as seen by combining materials with different hydrogen affinities. A 
quasi two-dimensional ensemble was realized, which resembled neither the bulk nor the surface properties of the material with respect to hydrogen absorption [31, 32]. Duda et al. reported in an early study in which the electronic structure of vanadium in a $\mathrm{Fe} / \mathrm{V}$ (001) superlattices exposed to a 100 torr hydrogen pressure in the gas cell was measured using soft X-ray emission spectroscopy [33]. It was found that the band broadening from band filling of the hydrogen electrons compensates the band narrowing effect expected from the $\mathrm{V}$ lattice expansion, while the hybridization of $\mathrm{H} 1 s$ and primarily $\mathrm{V} 3 d$ states only takes place at high binding energies but not to affect the main band.

When the sample were in the state of, e.g. liquid or gas at high pressure $(\leq 1 \mathrm{bar})$, the scheme of the in-situ cell with two membrane windows was no longer suitable. Instead a liquid cell with a single thin membrane window (typically a $100 \mathrm{~nm}$ thick silicon nitride, commercially available [34]) has been used to attain the compatibility of liquid samples with UHV conditions inside the experimental vacuum chamber [20, 21]. The incoming soft X-rays enter the liquid cell through the membrane window and the emitted X-rays exit through the same window. Transmission of $\mathrm{X}$-rays at the $\mathrm{C}$ and $\mathrm{O} K$-edges, and the Fe $L$-edge for a $100 \mathrm{~nm}$ thick silicon nitride window has been calculated to $c a$. $46 \%$, $66 \%$, and $82 \%$, respectively. Taking the limited photon attenuation length into account, we have performed soft X-ray spectroscopic studies of liquid water and solutions. The static liquid cell was able to withstand a pressure difference between the liquid (on one side) and UHV (on the other side) [35], which allowed experiments to be performed at the base pressure of less than $1 \times 10^{-9}$ torr. This experimental setup has facilitated the studies of hydrogen bonding in liquid water and also the mixture of liquid water and methanol $[20,21]$. Another study using this technique includes probing electronic structure of cobalt nanocrystals suspended in liquid solvent as a function of nanoparticle size using in-situ X-ray absorption spectroscopy, X-ray emission spectroscopy, and resonant X-ray Raman spectroscopy, where X-ray Raman features due to d-d and chargetransfer excitations of the Co nanocrystals and ligand molecules were identified. This study revealed the local symmetry of the surface of Co nanocrystals, which originates from a dynamic interaction between the Co nanocrystals and surfactant/solvent molecules. [36].

$\mathrm{X}$-ray induced sample damage is also a well-known problem in soft X-ray spectroscopic 
studies, especially for organic systems. Applying a continuous flow of liquid nitrogen or helium whilst measuring the sample are typical means to minimize/overcome beam induced sample damages. For a liquid sample, a flow liquid cell is used to avoid the problem by continuously refreshing the samples [37]. While the main architecture of a liquid flow cell is similar to that of the static cell, the liquid flow cell has a continuous flow of liquid sample to avoid X-ray induced sample damage. There have been many other developments on in-situ soft X-ray spectroscopy characterization at the ALS and other synchrotron facilities, for example, using various liquid flow scheme [38-45] or liquid jet. [46, 47] Further application of the liquid-cell scheme also allowed us to perform XAS experiments in the presence of liquid electrolytes under controlled electrochemical conditions $[48,49]$.
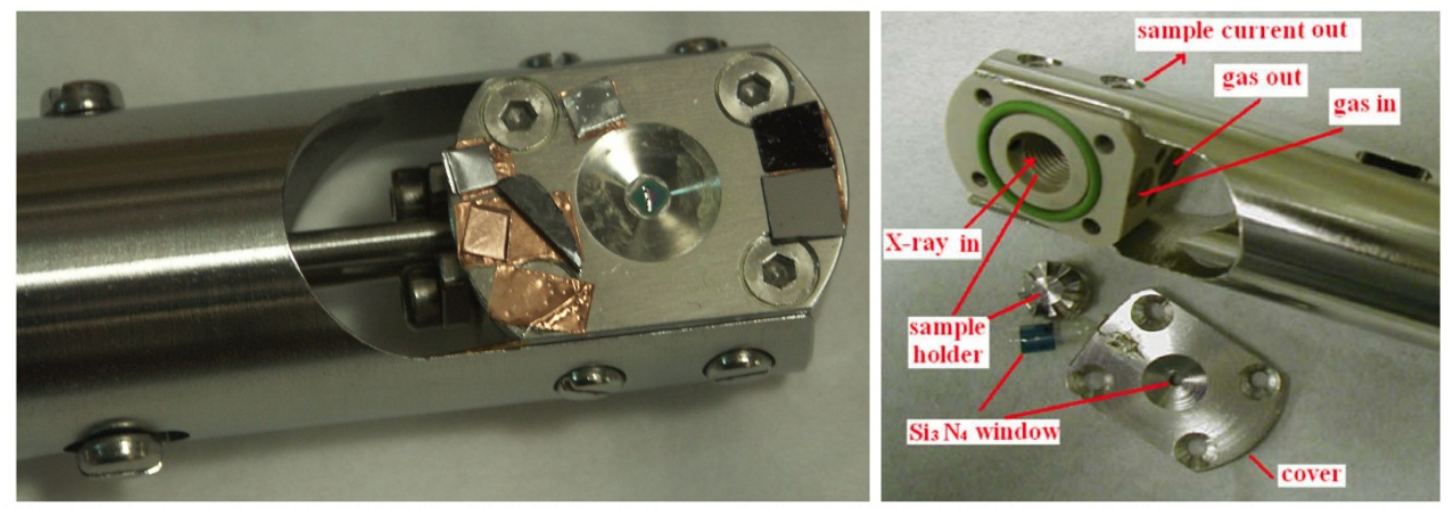

Figure 1. The assembled gas cell (left) and the central components of the gas cell (right), which was used in the studies of hydrogen molecular adsorption on SWNTs at room temperature under pressure up to 1 bar [ForsbergRSI07, ZhongCar10].

Figure 1 shows the first generation high pressure gas cell used on BL7.0.1 at the ALS. The design was similar to the earlier gas flow reaction cell built by Nordgren's group in Uppsala University [50]. The gas cell was constructed on a long supporting tubing mounted on a commercial manipulator with a sample holder inside the cell and two thin tubes for gas inlet and outlet with the O-ring seal to achieve a base pressure of $10^{-9}$ torr in the experimental vacuum chamber. The gas flow cell was used in the studies of $\mathrm{H}_{2}, \mathrm{~N}_{2}$ and He adsorption on the single walled carbon nanotubes (SWNTs) at room temperature in vacuum and ambient conditions (up to one atmospheric pressure) [51, 52]. It was 
shown that gas scattering contribution should be considered as a result of collisions between the gas molecules and SWNTs. It was shown how the occurrence of the scattering process and thereby gas collisions induced the changes in the electronic structure of SWNTs.

Another important function of the gas cell, the possibility to heat the sample in-situ is essential to perform any catalytic study. This feature was added to the third generation insitu cells and allowed in-situ spectroscopic measurements at elevated temperatures ( $>300$ ${ }^{\circ} \mathrm{C}$ ) and gas pressure up to 1 atmosphere at the same time [8, 53-55]. A typical $3^{\text {rd }}$ generation in-situ gas cell is presented in figure 2. This design was completed for experiments on BL7.0.1 and later modified to meet the need of e.g. a particular experiment or to prevent crosscontamination between the different experiments. These in-situ cells are currently being used on BL 6.3.1.2 and BL 8.0.1 at the ALS. The key components of the in-situ cells are shown in the figure 2: gas inlet and outlet (top), sample mounting (center), and the complete assembly (bottom). The cell has a cooling capability with liquid nitrogen and heating for high temperature catalytic reactions. Two coaxial Teflon-based sealing

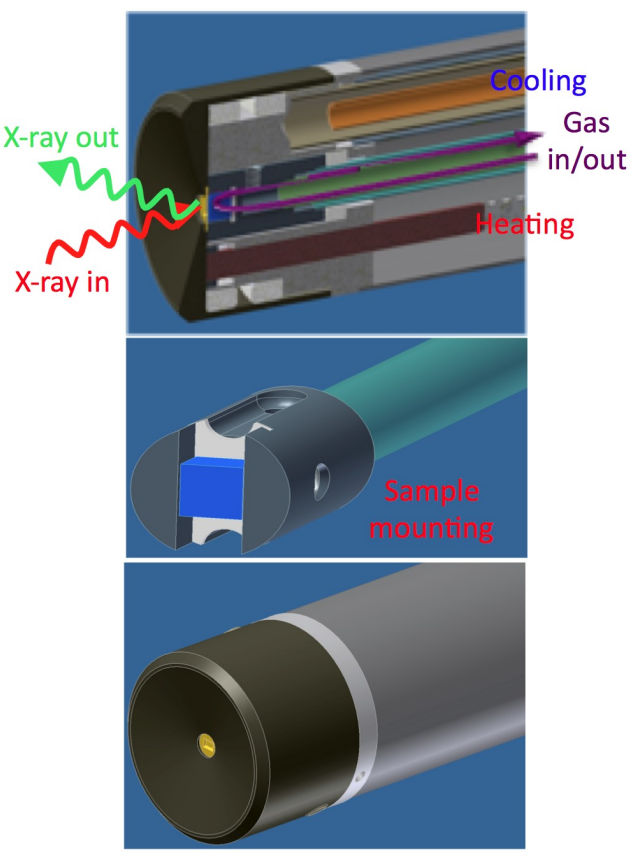

Figure 2. Soft $x$-rays can penetrate in and out through the $100 \mathrm{~nm}$ silicon nitride membrane window. Top: the gas inlet and outlet, cooling and heating; center: sample mounting; and bottom: the assembly. are used to allow high temperature $\left(>300^{\circ} \mathrm{C}\right)$ in operation, and a differential pumping stage was adopted to achieve a base pressure in the experimental vacuum chamber at $10^{-9}$ Torr. The sample mounting can be adjusted to host the samples of various thicknesses. The in-situ cells have been attached on a manipulator that allows the cell to be readily aligned to the incoming X-rays going through the silicon nitride membrane window. The long translation stroke of the manipulator allows for the extraction of the cell fully for sample preparation without disturbing the base pressure of the experimental vacuum 
chamber. The most recent development of gas cells for catalysis studies includes a goldcoated cell to prevent spurious reactions on the reaction chamber walls, and laser heating of the sample through a quartz window. This last feature makes possible to selectively heat the sample to a higher reaction temperature while keeping the walls at room temperature [56].

\section{In-situ soft X-ray spectroscopy studies}

It is widely known that the activity and selectivity of novel catalysts strongly depends on the principle characteristics of the nanocatalysts e.g. size, shape, composition and phase distribution (alloy or core-shell structures, etc). As previously mentioned, cobalt is a well-known catalyst for many catalytic applications, including the growth of carbon nanotubes, [57] the oxidation

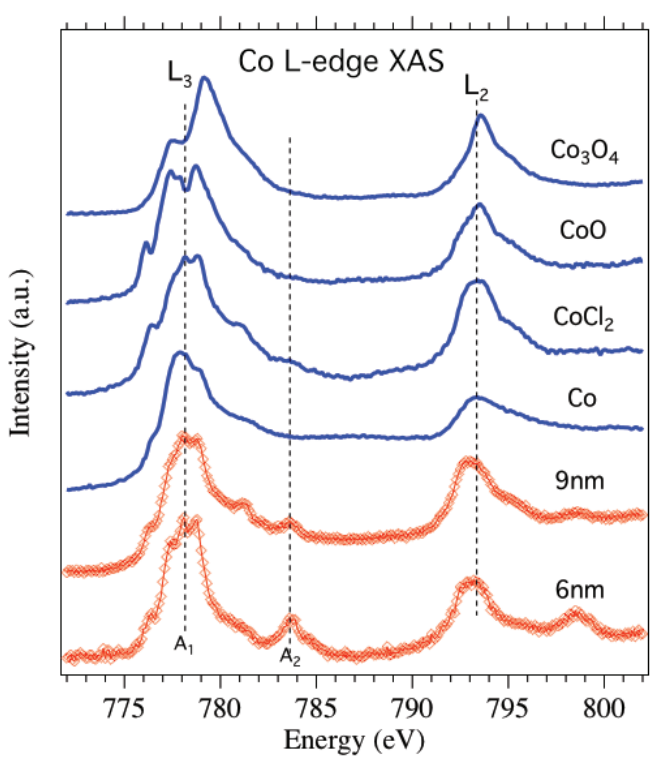

Figure 3. Co $2 \mathrm{p}$ X-ray absorption spectra of cobalt nanoparticles with diameters 6 and $9 \mathrm{~nm}$ in a 1,2dichlorobenzene liquid suspension. The top four spectra correspond to $\mathrm{Co}_{3} \mathrm{O}_{4}, \mathrm{CoO}, \mathrm{CoCl}_{2}$, and cobalt metal. [36]

of carbon monoxide, [58] the selective

reduction of nitrogen oxides [59] and the Fischer-Tropsch (FT) synthesis. [60, 61] In Fischer-Tropsch synthesis, it produces liquid hydrocarbons with high carbon efficiency. [62] Co nanocrystals display a wealth of size-dependent structural, magnetic, electronic, and catalytic properties. Using appropriate surfactants, Co nanocrystals can be grown with controlled shape and size, e.g. spheres or disks in a surfactant mixture. $[63,64]$ Insitu soft X-ray spectroscopy study of the electronic structure of the nanocrystals may provide the fundamental understanding of the growth and properties as a function of size and further the nature of the molecules bound to the surface. As Co nanocrystals are very reactive and oxidized if exposed to air, it is critical to characterize the nanoparticles in the growth environment so that their electronic and chemical properties can be monitored 
during the growth process and in the catalytic reactions. Thus, the very first XAS experiment was performed on the Co nanoparticle suspension in the static liquid cell [36]. Figure 3 presents Co 2p X-ray absorption spectra of cobalt nanoparticles (ca. 6 and $9 \mathrm{~nm}$ in diameter) in a 1,2-dichlorobenzene liquid suspension, along with Co metal, $\mathrm{CoCl}_{2}$, $\mathrm{Co}_{3} \mathrm{O}_{4}$, and $\mathrm{CoO}$ reference samples. The XAS spectra show two regions separated by $15.0 \mathrm{eV}$ originated from the core-level spin orbital splitting of the $2 \mathrm{p}_{3 / 2}$ and $2 \mathrm{p}_{1 / 2}$ orbitals. The high branching ratio of $\mathrm{L}_{2} /\left(\mathrm{L}_{2}+\mathrm{L}_{3}\right)$ in the reference spectra is a result of saturation effect from the total fluorescence-detection mode in XAS measurement, thus it cannot be used for quantitative analysis. However, the spectral shape of $\mathrm{L}_{3}$-edge can still be utilized for characterizing the Co oxidation states and crystalline structure. Recent studies have shown that XAS spectra obtained by partial fluorescence yield (PFY) detection can overcome the saturation effect related problems [65-69]. For that reason, only the $\mathrm{L}_{3}$-edge spectral profile of the XAS spectra was used for comparison in Figure 3, in which the Co nanoparticles (NPs) show a spectral shape close to that of the $\mathrm{Co}^{2+}$ oxidation state, suggesting a chemical interaction with the solvent molecules of 1,2-dichlorobenzene as the $\mathrm{L}_{3}$-edge XAS spectral profile is similar to that of $\mathrm{CoCl}_{2}$.

Later, Herranz et al. [8] reported the evolution in the Co $2 p$ state as a function of temperature in a Co NPs $(c a .10 \mathrm{~nm})$ catalyst system in which the NPs had been transferred from a Langmuir-Blodgett trough to a gold foil (figure 4). The SEM images in Figure $4 \mathrm{a}$ and $4 \mathrm{~b}$ of Co nanoparticles, taken before and after the catalytic reactions show no change for the size and dispersion. 


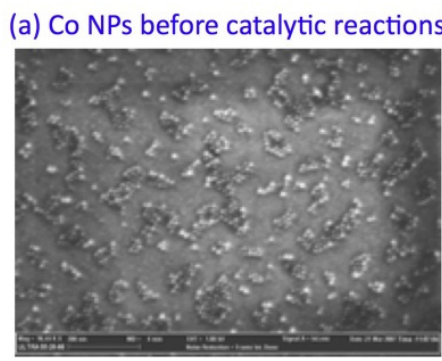

(b) Co NPs aftercatalytic reactions

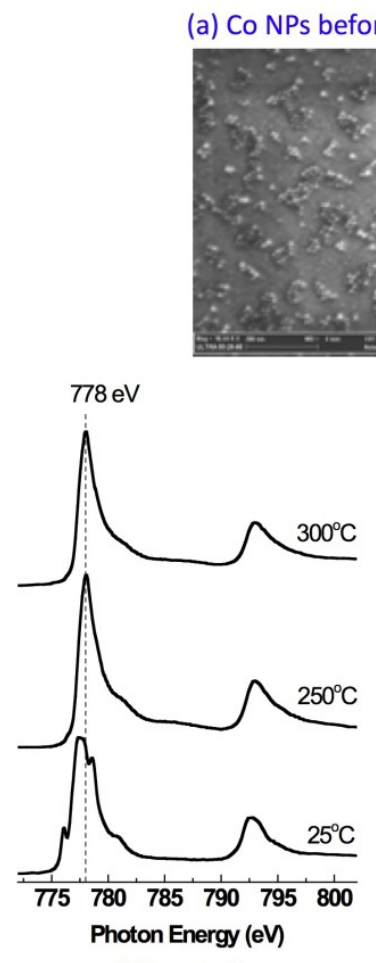

(c) Co Foil

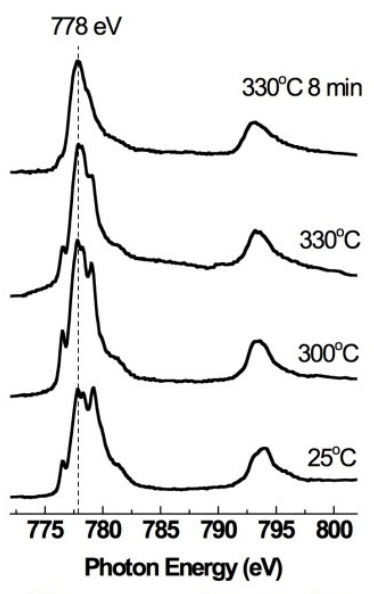

(d) as-prepared Co NPs (10 nm)
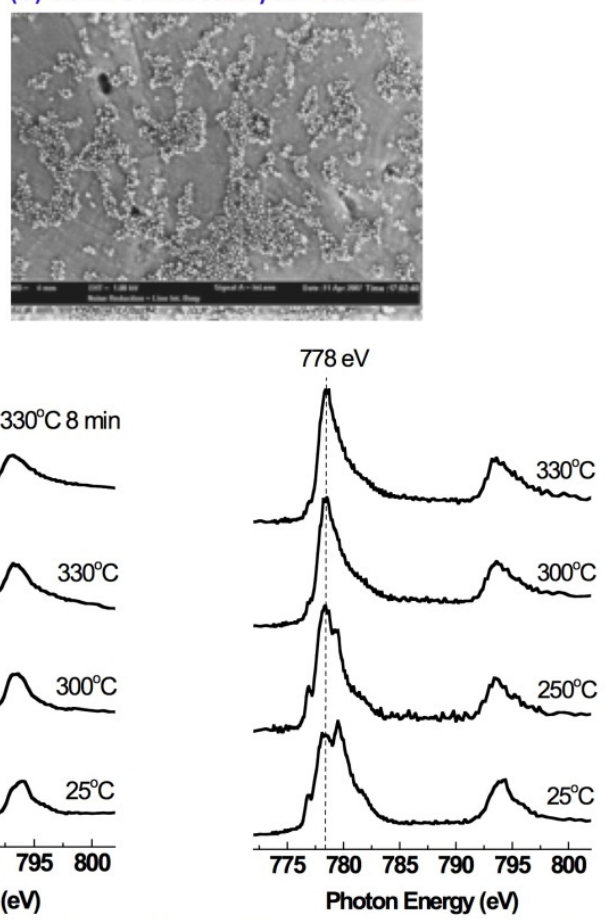

(e) pre-cleaned with $\mathrm{O}_{2}$ plasma in a $\mathrm{H}_{2}$ flow $(20 \mathrm{ml} / \mathrm{min})$

Figure 4. SEM images of Co NPs (a) prior to the catalytic reaction and (b) post catalytic reaction.(c)(e): Co L-edge spectra of Co foil and Co nanoparticles $(10 \mathrm{~nm})$ deposited over Au foils acquired in situ during reduction in a continuous flow of pure hydrogen at different temperatures. [HerranzJPC09]

In-situ XAS spectra were recorded under continuous $\mathrm{H}_{2}$ flow $(20 \mathrm{ml} / \mathrm{min})$ at 1 atm. pressure of He gas (figure 4). For Co NPs, the XAS spectra at room temperature correspond to that of $\mathrm{CoO}$, which contains $\mathrm{Co}^{2+}$ ions octahedrally coordinated with oxygen anions while Co was oxidized slightly on the surface. The presence of cobalt oxide is confirmed from the fine spectral features at ca. 776.5 and $780 \mathrm{eV}$. In the temperature range 250 to $330{ }^{\circ} \mathrm{C}$, the features in the X-ray absorption spectral feature resemble that for metallic Co, characterized by two asymmetric absorption white lines at 778 and $794 \mathrm{eV},[8]$ The surface oxidized Co NPs are reduced completely in 8 min after heating to $300^{\circ} \mathrm{C}$, or completely reduced instantly when heated up to $330^{\circ} \mathrm{C}$. For the samples after pre-treated with $\mathrm{O}_{2}$ plasma, the Co NPs could be reduced completely after a flash heating at a lower temperature of $300^{\circ} \mathrm{C}$.

In another study, pure cobalt and cobalt-platinum bimetallic nanoparticles were used to 
study the change of the chemical state in different reactant gases that are oxidizing (in $\mathrm{O}_{2}$ ) or reducing (in $\mathrm{H}_{2}$ ). Prior to introducing $\mathrm{H}_{2}$, Co L-edge XAS spectra were recorded at 1 bar He pressure at room temperature. The Co in the as-grown CoPt NPs is found to be at a more reduced state as compared with $\mathrm{Co}$ in the as-grown Co NPs. As $\mathrm{H}_{2}$ was introduced the Co became reduced completely in the CoPt NPs at $38^{\circ} \mathrm{C}$, while in the pure Co NPs the Co is only partially reduced to the metallic state at $250^{\circ} \mathrm{C}$. This difference demonstrates that Pt efficiently facilitates Co reduction as Pt plays a critical role in the process. Thus the in-situ XAS experiment demonstrates a significant impact of Pt on the reducibility of Co.

In summary, we have shown the development of in-situ cells for soft X-ray spectroscopic studies, and the application of the same on Co-based nano-catalysts in the catalytic reactions in the past decade on BL 7.0.1 at the ALS. The in-situ cells are now used on BL 6.3.1.2 and BL 8.0.1 after the termination of the BL 7.0.1 for further beamline development. A new beamline dedicated for in-situ/operando soft X-ray spectroscopy characterization using photon-in/photon-out and photon-in/electron-out spectroscopy, named Advanced Materials Beamline for Energy Research (AMBER), is currently under development at the ALS.

\section{Acknowledgement:}

The development of in-situ cells had its root in the gas cells and liquid cells originated from Nordgren's group in Uppsala University, in which the engineer, Carl-Johan Englund made the design and fabricated some of the in-situ cells. Many Nordgren's group members and BL7.0.1 users contributed significantly over the years. The in-situ cells made at the ALS have been benefited greatly from the engineers, Mark West and John Pepper. The Advanced Light Source is supported by the Director, Office of Science, Office of Basic Energy Sciences, of the U.S. Department of Energy under Contract No. DE-AC02-05CH11231. 


\section{References:}

[1] T. Okada, M. Kaneho, ed. Molecular Catalysts for Energy Conversion. Springer Series in Materials Science, Warlimont, Hans2009, Springer: Verlag Berlin Heidelberg.

[2] H. Schulz, "Short history and present trends of Fischer-Tropsch synthesis", Appl. Catal. A: Gen. 186 (1999) 3-12.

[3] M.E. Dry, “The Fischer-Tropsch process: 1950-2000”, Catal. Today 71 (2002) 227241.

[4] In this paper, in-situ characterization is meant to characterize the electronic structure of a given material during a process or event, such as a growth process of nanoparticles or catalysts in the catalytic reactions. On contrast, ex-situ characterization would be to characterize the electronic structure of a material after its growth or catalytic reaction.

[5] A. Ulman, "Formation and structure of self-assembled monolayers", Chem. Rev. 96 (1996) 1533-1554.

[6] Selim Alayoglu, Simon Beaumont, Fan Zheng, Vladimir V. Pushkarev, Haimei Zheng, Viacheslav Iablokov, Zhi 64, Jinghua Guo, Norbert Kruse, Gabor A. Somorjai, " $\mathrm{CO}_{2}$ Hydrogenation Studies on Co and CoPt Bimetallic Nanoparticles Under Reaction Conditions Using TEM, XPS and NEXAFS", Topics in Catalysis 54 (2011) 778-785.

[7] Sophie Carenco, Anders Tuxen, Mahati Chintapalli, Elzbieta Pach, Carlos Escudero, Trevor D. Ewers, Peng Jiang, Ferenc Borondics, Geoff Thornton, A. Paul Alivisatos, Hendrik Bluhm, Jinghua Guo, and Miquel Salmeron, "Dealloying of Cobalt from CuCo Nanoparticles under Syngas Exposure", J. Phys. Chem. C 117 (2013), 6259-6266.

[8] Tirma Herranz, Xingyi Deng, Andreu Cabot, Jinghua Guo, and Miquel Salmeron, "Influence of the Cobalt Particle Size in the CO Hydrogenation Reaction Studied by In Situ X-Ray Absorption Spectroscopy”, J. Phys. Chem. B 113 (2009) 10721-10727.

[9] Viacheslav Iablokov, Simon K. Beaumont, Selim Alayoglu, Vladimir V. Pushkarev, Colin Specht, Jinghua Guo, A. Paul Alivisatos, Norbert Kruse, and Gabor A. Somorjai, "Size-Controlled Model Co Nanoparticle Catalysts for $\mathrm{CO}_{2}$ Hydrogenation: Synthesis, Characterization, and Catalytic Reactions", Nano Lett. 12 (2012) 3091-3096.

[10] A. Tuxen, S. Carenco, M. Chintapalli, C. Chuang, C. Escudero, E. Pach, P. Jiang, F. Borondics, B. Beberwyck, A.P. Alivisatos, G. Thornton, W. Pong, J.-H. Guo, R. Perez, F. Besenbacher, and M. Salmeron, "Size-Dependent Dissociation of Carbon Monoxide on Cobalt Nanoparticles", J. Am. Chem. Soc. 135 (2013), 2273-2278.

[11] Chunrong Yin, Fan Zheng, Jinghua Guo, Gihan Kwon, Viktor Vajda, Hsien-Hau Wang, Sungsik Lee, Sönke Seifert, Janae DeBartolo, Randall E. Winans, and Stefan Vajda, "Composition of Oxidized Subnanometer Cobalt Clusters (4, 7 and 27 Co atoms): Size and Support Effects", J. Phys. Chem. A (2014). DOI: 10.1021/jp501817u

[12] D.F. Ogletree, H. Bluhm, G. Lebedev, C.S. Fadley, Z. Hussain, M. Salmeron, “A differentially pumped electrostatic lens system for photoemission studies in the millibar range”, Rev. Sci. Instr. 73 (2002) 3872. 
[13] A. Knop-Gericke, M. Haevecker, T. Schedel-Niedrig, and R. Schloegl, "Probing the electronic structure of an active catalyst surface under high-pressure reaction conditions: the oxidation of methanol over copper", Catal. Lett. 66 (2000) 215.

[14]A. Knop-Gericke, M. Haevecker, T. Schedel-Niedrig, and R. Schloegl, "Highpressure low-energy XAS: a new tool for probing reacting surfaces of heterogeneous catalysts", Top. Catal. 10 (2000) 187.

[15] I. Boettger, T. Schedel-Niedrig, O. Timpe, R. Gottschall, M. Haevecker, T. Ressler, and R. Schloegl, "Catalytic Methanol Oxidation over Copper: Observation of ReactionInduced Nanoscale Restructuring by Means of In Situ Time-Resolved X-ray Absorption Spectroscopy", Chem.-Eur. J. 6 (2000) 1870.

[16] A. Knop-Gericke, M. Haevecker, T. Schedel-Niedrig, and R. Schloegl, "Characterisation of active phases of a copper catalyst for methanol oxidation under reaction conditions: an in situ X-ray absorption spectroscopy study in the soft energy range", Top. Catal. 15 (2001) 27.

[17] P. Glans, K. Gunnelin, P. Skytt, J.-H. Guo, N. Wassdahl, J. Nordgren, H. Ågren, F. Kh. Gel'mukhanov, and T. Warwick, "Resonant X-ray Emission Spectroscopy of Molecular Oxygen", Phys. Rev. Lett. 76 (1996) 2448.

[18] P. Skytt, P. Glans, J.-H. Guo, K. Gunnelin, C. Såthe, J. Nordgren, F. Kh. Gel'mukhanov, A. Cesar, and H. Ågren, "Quenching of Symmetry Breaking in Resonant Inelastic X-ray Scattering by Detuned Excitation", Phys. Rev. Lett. 77 (1996) 5035-5038.

[19] Joseph Nordgren and Jinghua Guo, "Instrumentation for soft X-ray emission spectroscopy", J. Electron Spectrosc. Relat. Phenom. 110-111 (2000) 1-13.

[20] J.-H. Guo, Y. Luo, A. Augustsson, J.-E. Rubensson, C. Såthe, H. Ågren, H. Siegbahn, and J. Nordgren, "X-ray Emission Spectroscopy of Hydrogen Bonding and Electronic Structure of Liquid Water", Phys. Rev. Lett. 89 (2002) 137402.

[21] J.-H. Guo, Y. Luo, A. Augustsson, S. Kashtanov, J.-E. Rubensson, D. K. Shuh, H. Agren, and J. Nordgren, "The molecular structure of alcohol-water mixtures", Phys. Rev. Lett. 91 (2003) 157401.

[22] B.L. Henke, E.M. Gullikson, and J.C. Davis. X-ray interactions: photoabsorption, scattering, transmission, and reflection at E=50-30000 eV, Z =1-92, Atomic Data and Nuclear Data Tables Vol. 54 (1993) 181-342.

[23] P. Glans, P. Skytt, K. Gunnelin, J.-H. Guo, J. Nordgren, F. Gelmukhanov, A. Cesar, and H. Ågren, "Selectively-Excited X-ray Emission Spectra of $\mathrm{N}_{2}$ ", J. Electr. Spectr. Rel. Phenom. 82 (1996) 3.

[24] A. Cesar, F. Kh. Gel'mukhanov, Y. Luo, H. Ågren, P. Skytt, P. Glans, J.-H. Guo, K. Gunnelin, and J. Nordgren, "Resonant X-ray Scattering Beyond the Born-Oppenheimer Approximation: Symmetry Breaking in the Oxygen Resonant X-ray Emission of Carbon Dioxide", J. Chem. Physics 106 (1997) 3439.

[25] P. Skytt, P. Glans, K. Gunnelin, J.-H. Guo, J. Nordgren, Y. Luo, and H. Ågren, "Role of Screening and Angular Distributions in Resonant X-ray Emission Spectra of CO", Phys. Rev. A 55 (1997) 134-145. 
[26] P. Skytt, P. Glans, K. Gunnelin, J.-H. Guo, and J. Nordgren, "Lifetime Vibrational Interference Effects in Resonantly Excited X-ray Emission Spectra of CO", Phys. Rev. A 55 (1997) 146-154.

[27] J. Nordgren, P. Glans, K. Gunnelin, J.-H. Guo, P. Skytt, C. Såthe, N. Wassdahl, "Resonant Soft X-ray Fluorescence Spectra of Molecules", Appl. Phys. A 65 (1997) 97.

[28] K. Gunnelin, P. Glans, P. Skytt, J.-H. Guo, J. Nordgren, and H. Ågren, "Assigning X-ray absorption spectra by means of soft-x-ray emission spectroscopy", Phys. Rev. A 57 (1998) 864-872.

[29] Franz Hennies, Annette Pietzsch, Martin Berglund, Alexander Fohlisch, Thorsten Schmitt, Vladimir Strocov, Hans O. Karlsson, Joakim Andersson, and Jan-Erik Rubensson, "Resonant Inelastic Scattering Spectra of Free Molecules with Vibrational Resolution”, Phys. Rev. Lett. 104, 193002 (2010).

[30] A. Pietzsch, Y.-P. Sun, F. Hennies, Z. Rinkevicius, H. O. Karlsson, T. Schmitt, V. N. Strocov, J. Andersson, B. Kennedy, J. Schlappa, A. Fohlisch, J.-E. Rubensson, and F. Gel'mukhanov, Spatial Quantum Beats in Vibrational Resonant Inelastic Soft X-Ray Scattering at Dissociating States in Oxygen, Phys. Rev. Lett. 106, 153004 (2011).

[31] F. Stillesjoe, S. Olafsson, P. Isberg, and B. Hjoervarsson, "Thermodynamic properties of hydrogen in quasi-two-dimensional vanadium lattices”, J. Phys. Condens. Matter 7 (1995) 8139.

[32] G. Andersson, B. Hjoervarsson, and P. Isberg, "Influence of compressive biaxial strain on the hydrogen uptake of ultrathin single-crystal vanadium layers", Phys. Rev. B 55 (1997) 1774.

[33] L.-C. Duda, P. Isberg, P. H. Andersson, P. Skytt, B. Hjörvarsson, J.-H. Guo, C. Såthe, and J. Nordgren, "Hydrogen-Induced Changes of the Electronic States in Ultrathin Single-Crystal Vanadium Layers", Phys. Rev. B 55 (1997) 12914.

[34] Silson Ltd., JBJ Business Park, Northampton Road, Blisworth, Northampton, NN7 3DW, England (Email: peter.anastasi@silson.com).

[35] J.-H. Guo, P. Skytt, N. Wassdahl, and J. Nordgren, "In-situ and ex-situ characterization of thin films by soft X-ray emission spectroscopy", J. Electron Spectrosc. Relat. Phenom. 110-111 (2000) 41-67.

[36] H. 64, J.-H. Guo, Y. Yin, A. Augustsson, C. L. Dong, J. Nordgren, C. L. Chang, P. Alivisatos, G. Thornton, D. F. Ogletree, F. G. Requejo, F. de Groot, and M. Salmeron, "Electronic Structure of Cobalt Nanocrystals Suspended in Liquid", Nano Lett. 7 (2007) 1919-1922.

[37] Jinghua Guo, Tyler Tong, Lukas Svec, John Go, Chungli Dong, and Jau-Wern Chiou, "Soft-x-ray Spectroscopy Experiment of Liquids", JVST A 25 (2007), 1231-1233.

[38] S Myneni, Y Luo, L Å Näslund, M Cavalleri, L Ojamäe, H Ogasawara, A Pelmenschikov, Ph Wernet, P Väterlein, C Heske, Z Hussain, L G M Pettersson and A Nilsson, "Spectroscopic probing of local hydrogen-bonding structures in liquid water", J. Phys.: Condens. Matter 14 (2002) L213.

[39] C. Heske, U. Groh, O. Fuchs, L. Weinhardt, E. Umbach, Th. Schedel-Niedrig, Ch.- 
H. Fischer, M. Ch. Lux-Steiner, S. Zweigart, T. P. Niesen, F. Karg, J. D. Denlinger, B. Rude, C. Andrus, and F. Powell, "Monitoring chemical reactions at a liquid-solid interface: Water on $\mathrm{CuIn}(\mathrm{S}, \mathrm{Se})_{2}$ thin film solar cell absorbers", J. Chem. Phys. 119 (2003) 10467.

[40] M. Freiwald, S. Cramm, W. Eberhardt, and S. Eisebitt, "Soft X-ray absorption spectroscopy in liquid environments", J. Electron Spectrosc. Relat. Phenom. 137-140 (2004) 413.

[41] J. B. MacNaughton, M. V. Yablonskikh, A. H. Hunt, E. Z. Kurmaev, J. S. Lee, S. D. Wettig, and A. Moewes, "Solid versus solution: Examining the electronic structure of metallic DNA with soft x-ray spectroscopy”, Phys. Rev. B 74 (2006) 125101.

[42] Oliver Fuchs, Florian Maier, Lothar Weinhardt, Markus Weigand, Monika Blum, Michael Zharnikov, Jonathan Denlinger, Michael Grunze, Clemens Heske, Eberhard Umbach, "A liquid flow cell to study the electronic structure of liquids with soft X-rays", Nucl. Instr. and Meth. in Phys. Res. A 585, 172-177 (2008).

[43] M. Blum, L. Weinhardt, O. Fuchs, M. Bär, Y. Zhang, M. Weigand, S. Krause, S. Pookpanratana, T. Hofmann, W. Yang, J. D. Denlinger, E. Umbach, and C. Heske, "Solid and liquid spectroscopic analysis (SALSA) - a soft x-ray spectroscopy endstation with a novel flow-through liquid cell”, Rev. Sci. Instrum. 80, 123102 (2009).

[44] Masanari Nagasaka, Takaki Hatsui, Toshio Horigome, Yutaka Hamamura, Nobuhiro Kosugi, "Development of a liquid flow cell to measure soft X-ray absorption in transmission mode: A test for liquid water", J. Electron Spectrosc. Relat. Phenom. 177 (2010) 130-134.

[45] Takashi Tokushima, Yoshihisa Harada, Yuka Horikawa, Osamu Takahashi, Yasunori Senba, Haruhiko Ohashi, Lars G.M. Pettersson, Anders Nilsson, Shik Shin, "High resolution X-ray emission spectroscopy of water and its assignment based on two structural motifs", J. Electron Spectrosc. Relat. Phenom. 177 (2010) 192-205.

[46] K. R. Wilson, Matteo Cavalleri, B. S. Rude, Richard D. Schaller, A Nilsson, L G M Pettersson, N Goldman, Tony Catalano, J. D. Bozek and R. J. Saykally, "Characterization of hydrogen bond acceptor molecules at the water surface using near-edge x-ray absorption fine-structure spectroscopy and density functional theory", J. Phys.: Condens. Matter 14 (2002) L221.

[47] Emad F. Aziz, Stefan Eisebitt, Frank de Groot, Jau W. Chiou, Chungi Dong, and Jinghua Guo, Wolfgang Eberhardt, "Direct Contact vs. Solvent-shared Ion Pairs in $\mathrm{NiCl}_{2}$ Electrolytes Monitored by Multiplet Effects at the Ni(II) L-edge X-Ray Absorption", J. Phys. Chem. B 111 (2007) 4440-4445.

[48] P. Jiang, J.-L. Chen, F. Borondics, P.-A. Glans, M.W. West, C.-L. Chang, M. Salmeron, J. Guo, "In situ soft X-ray absorption spectroscopy investigation of electrochemical corrosion of copper in aqueous $\mathrm{NaHCO}_{3}$ solution", Electrochem. Commun. 12 (2010) 820.

[49] Timothy S. Arthur, Per-Anders Glans, Masaki Matsui, Ruigang Zhang, Biwu Ma, Jinghua Guo, "Mg deposition observed by in situ electrochemical Mg K-edge X-ray absorption spectroscopy”, Electrochem. Commun. 24, 43-46 (2012). 
[50] J. Forsberg, L.-C. Duda, A. Olsson, T. Schmitt, J. Andersson, J. Nordgren, J. Hedberg, C. Leygraf, T. Aastrup, D. Wallinder, J.-H. Guo, "System for in situ studies of atmospheric corrosion of metal films using soft x-ray spectroscopy and quartz crystal microbalance”, Rev. Sci. Instrum., 78 (2007) 083110.

[51] Jun Zhong, Jinghua Guo, Bin Gao, Jau-Wern Chiou, Jiong Li, Wangsheng Chu, Way-Faung Pong, Chinglin Chang, Yi Luo, Ziyu Wu, "Understanding the scattering mechanism of single-walled carbon nanotube based gas sensors", Carbon 48 (2010) 1970-1976.

[52] Jun Zhong, Jauwern Chiou, Chungli Dong, Per-Anders Glans, Way-Faung Pong, Chinglin Chang, Ziyu Wu, and Jinghua Guo, "Interfacial interaction of gas molecules and single-walled carbon nanotubes”, Appl. Phys. Lett. 100 (2012) 201605.

[53] A. Knop-Gericke, M. Haevecker, Th. Neisius, Th. Schedel-Niedrig, "New experimental technique: X-ray absorption spectroscopy detector for in situ studies in the soft X-ray range $(250 \mathrm{eV} \leqslant \mathrm{hv} \leqslant 1000 \mathrm{eV})$ under reaction conditions", Nucl. Instrum. Methods A 406 (1998) 311.

[54] M. Hävecker, A. Knop-Gericke, Th. Schedel-Niedrig, "High-pressure soft X-ray absorption spectroscopy: application of a new in situ spectroscopic method in catalysis research", Applied Surface Science 142 (1999) 438.

[55] F. Zheng, F., S. Alayoglu, J. Guo, V. Pushkarev, Y. Li, P.-A. Glans, J.-L. Chen, G. Somorjai, "In-situ X-ray Absorption Study of Evolution of Oxidation States and Structure of Cobalt in Co and CoPt Bimetallic Nanoparticles $(4 \mathrm{~nm})$ under Reducing $\left(\mathrm{H}_{2}\right)$ and Oxidizing $\left(\mathrm{O}_{2}\right)$ Environments", Nano Letters 11 (2011) 847.

[56] Carlos Escudero, Peng Jiang, Elzbieta Pach, Ferenc Borondics, Mark W. West, Anders Tuxen, Mahati Chintapalli, Sophie Carenco, Jinghua Guo, Miquel Salmeron. J. of Synchrotron Radiation. 20 (2013) 504-508.

[57] E. Terrado, Mater. Sci. Eng. C 26 (2006) 1185.

[58] W. H. Yang, M. H. Kim, S. H. Ham, "Effect of calcination temperature on the lowtemperature oxidation of $\mathrm{CO}$ over $\mathrm{CoO}_{x} / \mathrm{TiO}_{2}$ catalysts", Catal. Today 123 (2007) 94.

[59] J. N. Armor, "Catalytic reduction of nitrogen oxides with methane in the presence of excess oxygen: A review”, Catal. Today 26 (1995) 147.

[60] M. E. Dry, Catalysis: Science and Technologies; Anderson, J. R., Boudart, M., Eds.; Springer-Verlag: Berlin, 1981; Vol. 1 p 159.

[61] A. Y. Khodakov, W. Chu, P. Fongarland, "Advances in the development of novel cobalt Fischer-Tropsch catalysts for synthesis of long-chain hydrocarbons and clean fuels", Chem. Rev. 107 (2007) 1692.

[62] G. Jacobs, T. Das, Y. Zhang, J. Li, G. Racoillet, B. H. Davis, "Fischer-Tropsch synthesis: support, loading, and promoter effects on the reducibility of cobalt catalysts", Appl. Catal. A: Gen. 233 (2002) 263.

[63] V. F. Puntes, K. M. Krishnan, A. P. Alivisatos, Science 291, 2115-2117 (2001).

[64] V. F. Puntes, P. Gorostiza, D. M. Aruguete, N. G. Bastus, A. P. Alivisatos, Nat. 
Mater. 3, 263-268 (2004).

[65] A. J. Achkar, T. Z. Regier, E. J. Monkman, K. M. Shen, and D. G. Hawthorn, "Determination of total x-ray absorption coefficient using non-resonant x-ray emission", Nature Sci. Rep. 1 (2011) 182.

[66] Reshmi Kurian, Kristjan Kunnus, PhilippeWernet, Sergei M Butorin, Pieter Glatzel and Frank M F de Groot, "Intrinsic deviations in fluorescence yield detected x-ray absorption spectroscopy: the case of the transition metal $\mathrm{L}_{2,3}$ edges", J. Phys.: Condens. Matter 24 (2012) 452201.

[67] T. Z. Regier, A. J. Achkar, D. Peak, J. S. Tse and D. G. Hawthorn, "Dark channel fluorescence observations result from concentration effects rather than solvent-solute charge transfer", Nature Chem. 4 (2012) 765.

[68] Frank M. F. de Groot, "Dips and peaks in fluorescence yield X-ray absorption are due to state-dependent decay", Nature Chem. 4 (2012) 766.

[69] Emad F. Aziz, Kathrin M. Lange, Sébastien Bonhommeau and Majed Chergui, "Reply to 'Dark channel fluorescence...' and 'Dips and peaks...' ", Nature Chem. 4 (2012) 767. 